\title{
Internet of Things (IoT) based home automation using ARM7
}

\author{
Pavithra G S \\ ${ }^{1}$ Assistant Professor, \\ Department of ECE, \\ Sai Vidya Institute of Technology, \\ Bangalore, India
}

\author{
Harshitha $\mathrm{K}^{2}$, Mohana $\mathrm{H} \mathrm{C}^{3}$, Amrutha $\mathrm{K} \mathrm{C}^{4}$ \\ $2,3 \& 4$ Student, \\ Department of ECE, \\ Sai Vidya Institute of Technology, Bangalore, India
}

\begin{abstract}
This project investigates the potential of Home Control and security which is the aim of the Home Automation Systems in near future. Automation is a system that plays a significant role in the ubiquitous economics. This paper represents a low cost, flexible and standalone home automation system. We can control home appliances from outdoor also from indoor locations.

The analysis and implementation of the home automation technology using Global System for Mobile Communication (GSM) modem to control home appliance such as light, conditional system, and security system via Short Message Service (SMS) text messages is presented in this Project. We also describe the implementation of a small-scale prototype intrusion detection and web service alarm design using ARM microprocessor. This intrusion detection system will be integrated wirelessly to the home Wi-FI system and could initiate an email to the respective authority. A computer screen or a Smartphone can be used to monitor the Main control Unit (MCU). A very simplistic Graphical User Interface (GUI) is used which can be easily understandable for the target user. To make the data easily available to the user, this GUI will be implemented on the webpage.
\end{abstract}

Keywords-Home Automation, GSM modem, Security System, ARM microprocessor, Internet of Things)

\section{INTRODUCTION}

Just conceive that, how beneficial it will to be able switch on our air condition half hour before we reach our home on summer time. When we leave our home for some work without realizing that some appliances such as fans, air conditioners, and lights are on; then by using our mobile phone or internet, we are able to turn off power to those devices. It will be even more useful if the system detects unauthorized movement in the house and alerts us or sends messages on our mobile phones or we can know the status of our house anytime.

Such systems provide security from natural, incidental, intended, unintended, accidental and human made problems by continuously monitoring homes with different sensory systems like motion, smoke, gas, temperature, glass break or door break detectors and fire alarm systems.

Security is a big challenge everywhere because thefts are increasing day by day owing to the unsafe and insecure security systems in homes, commercial complexes and industries. Several conventional technologies are available to keep home properties safe from intruders, but most common smart home security systems work on wireless GSM communication and micro devices in terms of microcontrollers and multi-core or GPU based microprocessors.

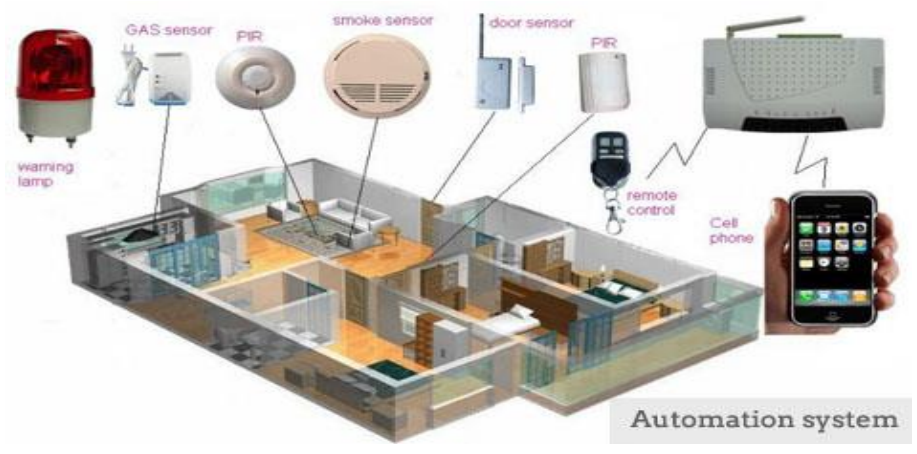

Fig.1 Home Automation and Security System

Security is one thing that is very influential in today life; everyone needs security guarantees when they work. Like health, security is an important aspect in life. Hence, various kinds of development in the technology field is designed to provide security at all times to protect them assets and privacy.

In addition to the course with the application of security system, it can reduce the crime rate in the society especially the crime of theft at home.

In this project, the home appliances that are connected to the Main Control Unit (MCU) will be controlled wirelessly with the help of a Smartphone or a web page. For simplicity, a Graphical User Interface (GUI) will be made available to the users so that the user can monitor the appliance as per his wish. This GUI must be implemented on the web page so that the user can easily access it anytime and anywhere.

The intrusion detection system will be integrated wirelessly to the home Wi-Fi system and could initiate an email to the respective authority. This web service will retrieve the image from ARM microprocessor and trigger an SMTP email service call. Then the intrusion event image is communicated to the authoritative person via email. In the end, the authority can analyze for false alarms and seek 911 helpline for immediate theft-response. The additional advantages of this application is the back-up image of intrusion event, which plays a key role for home-owners, police, and insurance agencies by confirming the intrusion event and could assist in identification of the intruder.

This system will reduce the overall power consumption thus saving the energy as the user can enable or disable the appliances according to his needs. 


\section{SYSTEM DESCRIPTION}

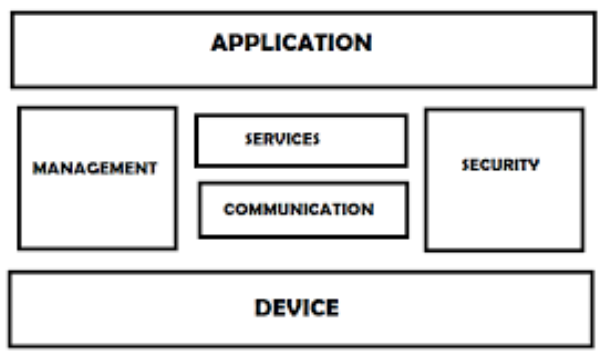

Fig 2 : Basic block diagram of home automation

The home automation system that works on $\mathrm{Wi}-\mathrm{Fi}$ technology. The main components of this technology are we server, hardware interface mode and sensors. Where, as the web server which is system core that controls various function in system and even monitors the users. Hardware interface module consists a Wi-Fi shield, 3 input alarms and 3 output actuators. Sensors which act to provide appropriate interface of home automation system.

The system is designed basically from flexibility point of view for user, instead of commercial purpose of home automation system. The user uses this technology to login to the server web-based application. The web server is connected to the internet which uses compatible web browser. This application can also be designed and developed in android system.

An interface card is developed to establish communication between the user, server, raspberry pi card and home appliances. We need to install the application on a smartphone, a web server and a raspberry pi card to establish the control over the windows. Android application gives the commands to raspberry pi card. An interface card is used for sending signals between actuator sensors and raspberry pi card.

Cloud based home appliance monitoring and controlling system. Designing and implementation of a home gateway for collecting metadata from appliances and then sent to cloudbased data server to store on Handoop Distribution file system (HDFS) process them using MAP reduce and use it to provide a monitoring function to remote user.

Wi-Fi technology can be implemented by studying the subject e-mail and the algorithm using raspberry pi. Raspberry pi is been a powerful, economic and a efficient platform for implementation of smart home automation. This method of implementation is better than others in various ways. For example, through dual tone multi frequency (DTMF) technology, the call traffic cause major disadvantage, which is not the case in presented idea. In web server based on home automation, the design of server and memory space required is removed by this method. In this case use LED's are used to indicate the switching action. System is interactive and very efficient purpose.

The home network which keeps track of the appliances and sensors and transmit data to cloud-based data server which manages the data and gives services for user by sending or transmitting data and receiving commands by the user from mobile. The system designed for home automation has good characteristics and modularity with low power usage. Its efficient way in terms of cost.

Android platform controlled and monitored by application developed. The sensors and actuators/relays are directly interfaced to the main controller. It is designed for system such as lighting, heating, air condition, security and fire fir detection etc. with email notification. This can make life way simpler and reduce work for us.

\section{METHODOLOGY}

The microcontroller arm7 is the heart of the project in which it controls the operation of the entire project. The home automation by using arm 7 microcontroller is basically done using different types of sensors in which it constantly census information with the help of these sensors and it controls different home appliances with the help of activators. This project consists of five types of sensors which are mainly
1. LPG sensor
2. Temperature sensor
3. Humidity sensor
4. IR sensor
5. LDR sensor

Each sensors have five relays in order to Control the different types of Home appliances which are

1. Light

2. Air conditioner or cooler

3. Fan

4. Geyser

In this project, the communication protocol which is used to communicate with the user is the GSM. The presence of the toggle switch helps to send information over a GSM modem when it is pressed.

The microcontroller helps to keep a check on the analog input pins providing the data from temperature sensor, humidity sensor and the LDR sensor.

The LM35 temperature sensor helps to obtain the reading of temperature through a ADC1 channel 4 of the microcontroller only when the temperature of the room goes beyond 40 degree in Celsius. The fan is on with the help of the relay driver circuit connected to $\mathrm{P} 0.30$.

The humidity sensor here helps to obtain the reading of the humidity through ADC1 channel 3 of the microcontroller only if the humidity goes beyond $70 \%$. Here the cooler or the air conditioner is on with the help of the relay driver circuit connected to P0.29.

The LDR sensor helps to sense the light and is usually placed near the window. The bulb near the window is switched on only when it is dark.

The microcontroller keeps on checking the digital input of the IR and the LPG sensor through the particular pins P0.17 and $\mathrm{P} 0.18$ respectively. 
The IR sensor is placed near the door so that when a person opens the door, the IR sensor sends a digital output one to the controller through the pin P0.17. Once when there is a detection found, the controller activates the CCTV camera and at the same time a message is sent to the particular mobile number stored in the controller through the GSM modem.

If there is any old person present or residing at the house and in case if that person needs any help in the absence of the members at the house, an alarm can be used. In this project there is a switch in which when it is pressed, a message will be sent through the modem to the person whose mobile number is stored in the microcontroller which informs that the person who is sending the message is seeking for help.

In this project, we can control the CCTV camera by a message through the modem in which when a message is been sent to a sim installed in the GSM modem saying Cam on\$, the message received by the modem is transferred into the controller. The microcontroller decodes the message and activates the camera through the relay connected to $\mathrm{P} 0.31$. In the case of Cam off\$, this message is received by the modem and is transferred to the microcontroller. The controller decodes the message and deactivates the camera through the relay connected to $\mathrm{P} 0.31$.

When there is no one present at the house but when a member wants to on the geyser, we can control this action through the modem in which when a message is sent to the sim installed inside the GSM modem indicating geyser on $\$$, the message received by the modem is transferred to controller. The microcontroller decodes the message and it activates the geyser through the relay connected to P0.25.

In the case of deactivating the geyser, we can send a message to the sim we which has been installed inside the modem geyser off\$. This message from the GSM modem is sent to the microcontroller in which it decodes the message and switches off the geyser through a relay connected to P0.25.

The LPG sensor is usually installed at the kitchen in which it detects any leakage of gas, if any, it sends a digital output to the controller through $\mathrm{P} 0.18$ pin. Once when it is detected, the microcontroller activates the buzzer through $\mathrm{P} 0.23$ pin of the controller and also a message will be sent to the registered mobile number on the controller through the modem.

In the case of the mobile user end, the message sent from the project which are received is the number which has been fed to the microcontroller of the installed project. The message sent via the standard format can be easily read by the mobile as another message. The messages usually received are

1. If there is a person being detected through the IR sensor when there is any disturbance near or at the door.

2. Need of help when an old person at home seeks any kind of help.
3. If there is any LPG gas leakage in which it is been detected by the gas sensor which detects the gas leakage.

In this project, we have 4 particular commands in the form of a standard format like the message sent to the mobile number of the sim installed in the home automation project received by the modem and is decoded. The respective geyser and the camera is on and off. The commands are

1. Cam on $\$$ and Cam off $\$$ for activating/on and deactivating/off of the camera respectively.

2. Geyser on\$ and Geyser off\$ for activating/on and deactivating/off of the geyser respectively.

\section{ACKNOWLEDGMENT (Heading 5)}

The preferred spelling of the word "acknowledgment" in America is without an "e" after the "g". Avoid the stilted expression "one of us (R. B. G.) thanks ...". Instead, try "R. B. G. thanks...". Put sponsor acknowledgments in the unnumbered footnote on the first page.

\section{REFERENCES}

The template will number citations consecutively within brackets [1]. The sentence punctuation follows the bracket [2]. Refer simply to the reference number, as in [3] - do not use "Ref. [3]" or "reference [3]" except at the beginning of a sentence: "Reference [3] was the first ..."

Number footnotes separately in superscripts. Place the actual footnote at the bottom of the column in which it was cited. Do not put footnotes in the abstract or reference list. Use letters for table footnotes.

Unless there are six authors or more give all authors' names; do not use "et al.". Papers that have not been published, even if they have been submitted for publication, should be cited as "unpublished" [4]. Papers that have been accepted for publication should be cited as "in press" [5]. Capitalize only the first word in a paper title, except for proper nouns and element symbols.

For papers published in translation journals, please give the English citation first, followed by the original foreignlanguage citation [6].

[1] G. Eason, B. Noble, and I. N. Sneddon, "On certain integrals of Lipschitz-Hankel type involving products of Bessel functions," Phil. Trans. Roy. Soc. London, vol. A247, pp. 529-551, April 1955. (references)

[2] J. Clerk Maxwell, A Treatise on Electricity and Magnetism, 3rd ed., vol.

[3] 2. Oxford: Clarendon, 1892, pp.68-73.

[4] I. S. Jacobs and C. P. Bean, "Fine particles, thin films and exchange anisotropy,” in Magnetism, vol. III, G. T. Rado and H. Suhl, Eds. New York: Academic, 1963, pp. 271-350.

[5] K. Elissa, "Title of paper if known," unpublished.

[6] R. Nicole, "Title of paper with only first word capitalized," J. Name Stand. Abbrev., in press.

[7] Y. Yorozu, M. Hirano, K. Oka, and Y. Tagawa, "Electron spectroscopy studies on magneto-optical media and plastic substrate interface," IEEE Transl. J. Magn. Japan, vol. 2, pp. 740-741, August 1987 [Digests 9th Annual Conf. Magnetics Japan, p. 301, 1982].

[8] M. Young, The Technical Writer's Handbook. Mill Valley, CA University Science, 1989. 
IEEE conference templates contain guidance text for composing and formatting conference papers. Please ensure that all template text is removed from your

We suggest that you use a text box to insert a graphic (which is ideally a 300 dpi TIFF or EPS file, with all fonts embedded) because, in an MSW document, this method is somewhat more stable than directly inserting a picture.

To have non-visible rules on your frame, use the MSWord "Format" pull-down menu, select Text Box > Colors and Lines to choose No Fill and No Line. conference paper prior to submission to the conference. Failure to remove template text from your paper may result in your paper not being published. 